\title{
ON THE POSSIBLE EXISTENCE OF NEW FERMIONIC DEGREES OF FREEDOM IN
}

$$
D=6
$$

\section{P. MIŠKINIS}

Department of Physics, Faculty of Fundamental Sciences, Vilnius Gediminas Technical University

Saulètekio Ave 11, LT-2040 Vilnius, Lithuania

E-mail: paulius.miskinis@fm.vtu.lt

Received July 18, 2002

\begin{abstract}
The bispinors formed by quaternion in $D=6$ dimensional space-time are proposed to be treated as new fermionic fields. The gauge nonabelian field is formulated by the quaternions. A new kind of physical object, an extended relativistic quaternionic membrane in $D=6$, is discussed.

Key words: fermionic fields, nonabelian field, quaternions, extended object, relativistic quaternionic membrane
\end{abstract}

\section{INTRODUCTION}

Global supersymmetry, an invariance regarding reparametrisations, and local fermionic gauge transformations predetermine the critical dimensions of the space-time $D=3,4,6$ and 10 , in which the existence of the classical covariant Green-Schwartz superstring is possible $[9 ; 16]$.

The number of independent parameters of the local fermionic gauge invariance in these critical dimensions equals $1,2,4$ and 8 , respectively. These numbers are equal to the dimensions of four algebras with the division of $\mathbb{R}, \mathbb{C}, \mathbb{H}$ and $\mathbb{O}$ real and complex numbers, quaternion and octonions. Besides, the existence of a series of isomorphisms, $\overline{S O}(2,1) \simeq S L(2, \mathbb{R}), \overline{S O}(3,1) \simeq S L(2, \mathbb{C})$, $\overline{S O}(5,1) \simeq S L(2, \mathbb{H})$ and $\overline{S O}(9,1) \simeq S L(2, \mathbb{O})[11 ; 18]$, naturally leads to the idea to expand the concept of spinor and to describe the properties of superstring in terms of algebras with division $[7 ; 8 ; 10 ; 15]$.

Usually the quaternionic formalism is applied as a mathematical tool for 
consideration of geometric or algebraic constructions with additional symmetries, for instance to description of supersymmetric spaces $[2 ; 3 ; 19]$, for reformulation of quantum mechanics $[13 ; 17]$, eigenvalues of differential operators [12], etc.

The aim of the present work is to point out the possibility in principle to treat the quaternion-induced spinors in $D=6$ not as a mathematical means of describing the known degrees of freedom, but as the absolutely new fermionic fields. This idea naturally leads to the extended relativistic objects like $p$ branes with quaternionic degrees of freedom.

\section{SPINORS}

Despite $\overline{S O}(5,1) \simeq S L(2, \mathbb{H})$ we will reject the total change of theory by introducing the quaternionic Gilbert space in $D=6$.

We will employ a matrix presentation of an arbitrary quaternion $q=a+$ $b e_{1}+c e_{2}+d e_{3}$, where $e_{i}=\varepsilon_{i j k} e_{j} e_{k}$ and $e_{i}^{2}=-1$ :

$$
q=\left(\begin{array}{cc}
a+b i & c+d i \\
-c+d i & a-b i
\end{array}\right) \equiv\left(\begin{array}{cc}
u & v \\
-v^{*} & u^{*}
\end{array}\right)
$$

$(a, b, c, d \in \mathbb{R} ; u, v \in \mathbb{C})$.

Then from the group $S L(2, \mathbb{H})$ we can move to a certain isomorphic group $\tilde{G}(4, \mathbb{C})$ above the field $\mathbb{C}$ of complex numbers:

$$
\tilde{G}(4, \mathbb{C}) \approx\left(\begin{array}{cccc}
u_{1} & v_{1} & u_{2} & v_{2} \\
-v_{1}^{*} & u_{1}^{*} & -v_{2}^{*} & u_{2}^{*} \\
u_{3} & v_{3} & u_{4} & v_{4} \\
-v_{3}^{*} & u_{3}^{*} & -v_{4}^{*} & u_{4}^{*}
\end{array}\right)
$$

bound by the requirement $\exp [\operatorname{Re}(\operatorname{tr} \ln \tilde{G})]=1$, which coincides with the condition of unimodularity for $S L(2, \mathbb{H})[3]$.

Now, any $\tilde{G}(4, \mathbb{C})$ Hermitian covector $X_{\mu}$ can be written in the form

$$
X_{\mathrm{A} \dot{A}}=\sigma_{A \dot{A}}^{\mu} X_{\mu} ; \quad \tilde{X}^{\dot{A} A}=\varepsilon^{\dot{A} \dot{B}}(X)_{\dot{B} B} \varepsilon^{A B}=\left(\tilde{\sigma}^{\mu}\right)^{\dot{A} A} X_{\mu}
$$

where

$$
\varepsilon^{A B}=\left(\begin{array}{rr}
0 & I \\
-I & 0
\end{array}\right)=-\varepsilon^{\dot{A} \dot{B}}
$$

$I$ is the unity matrix $, \sigma_{A \dot{A}}^{\mu}, \tilde{\sigma}^{\mu \dot{A} A}$ is a quaternion-induced analogue of the Pauli matrices for which an analogous property is valid:

$$
\begin{gathered}
\sigma_{A \dot{A}}^{\mu} \tilde{\sigma}^{\nu \dot{A} A}+\sigma_{A \dot{A}}^{\nu} \sigma^{\mu \dot{A} A}=2 g^{\mu \nu}, \\
\text { sign } g^{\mu \nu}=(+,-,-,-,-,-) .
\end{gathered}
$$


On the possible existence of new fermionic degrees of freedom in $D=6157$

Specifically, these matrices can have the following representation:

$$
\begin{aligned}
\sigma^{0} & =\left(\begin{array}{cccc}
1 & 0 & 0 & 0 \\
0 & 1 & 0 & 0 \\
0 & 0 & 1 & 0 \\
0 & 0 & 0 & 1
\end{array}\right), & \sigma^{1} & =\left(\begin{array}{cccc}
0 & 0 & 0 & 1 \\
0 & 0 & 1 & 0 \\
0 & 1 & 0 & 0 \\
1 & 0 & 0 & 0
\end{array}\right), \\
\sigma^{2} & =\left(\begin{array}{cccc}
0 & 0 & -i & 0 \\
0 & 0 & 0 & i \\
i & 0 & 0 & 0 \\
0 & -i & 0 & 0
\end{array}\right), & \sigma^{3} & =\left(\begin{array}{cccc}
0 & 0 & 0 & -1 \\
0 & 0 & 1 & 0 \\
0 & 1 & 0 & 0 \\
-1 & 0 & 0 & 0
\end{array}\right), \\
\sigma^{4} & =\left(\begin{array}{cccc}
0 & 0 & 0 & -i \\
0 & 0 & -i & 0 \\
0 & i & 0 & 0 \\
i & 0 & 0 & 0
\end{array}\right), & \sigma^{5} & =\left(\begin{array}{cccc}
1 & 0 & 0 & 0 \\
0 & 1 & 0 & 0 \\
0 & 0 & -1 & 0 \\
0 & 0 & 0 & -1
\end{array}\right) ;
\end{aligned}
$$

$\tilde{\sigma}^{0}=\sigma^{0}, \tilde{\sigma}^{m}=-\sigma^{m}$ for $m=1, \ldots, 5$.

The quaternion-induced covariant $\varphi_{A a}$ and contravariant dotted spinor $\chi_{a}^{\dot{A}}$ are $4 \times 2$ matrices:

$$
\begin{gathered}
\varphi_{A a}=\left(\begin{array}{rr}
u_{1} & v_{1} \\
-v_{1}^{*} & u_{1}^{*} \\
u_{2} & v_{2} \\
-v_{2}^{*} & u_{2}^{*}
\end{array}\right), \quad \chi_{a}^{\dot{A}}=\left(\begin{array}{cc}
u^{1} & v^{1} \\
-v^{1 *} & u^{1 *} \\
u^{2} & v^{2} \\
-v^{2 *} & u^{2 *}
\end{array}\right) ; \\
\varphi_{A a}^{\prime}=a_{A}^{B} \varphi_{B a}, \quad \chi^{\dot{A}}=\left(a^{+}\right)_{\dot{B}}^{-1 \dot{A}} X_{a}^{\dot{B}},
\end{gathered}
$$

where $a_{A}^{B} \in \tilde{G}(4, \mathbb{C}), \quad A=1, \ldots, 4 ; \quad \dot{A}=\dot{1}, \ldots, \dot{4} ; \quad a=1,2 ; u_{i}, v_{i} \in \mathbb{C}$.

\section{THE DIRAC EQUATION}

The system of two $\tilde{G}(4, \mathbb{C}) \cong S L(2, \mathbb{H})$ invariant equations in $D=6$

$$
\left\{\begin{array}{l}
i\left(\sigma^{\mu}\right)_{A \dot{A}} \partial_{\mu} \chi_{a}^{\dot{A}}-m \varphi_{A a}=0, \\
i\left(\tilde{\sigma}^{\mu}\right)^{\dot{A} A} \partial_{\mu} \varphi_{A a}-m \chi_{a}^{\dot{A}}=0,
\end{array}\right.
$$

for the covariant and contravariant dotted matrix spinors can be contracted into one equation by introducing the bispinor

$$
\psi_{\alpha a}=\left(\begin{array}{c}
\varphi_{A a} \\
\chi_{a}^{\dot{A}}
\end{array}\right)
$$

where $\alpha=(A, \dot{A})=1, \ldots 8 ; a=1,2$ and an analogue of Dirac matrices 


$$
\Gamma_{\alpha}^{\mu \beta}=\left(\begin{array}{cc}
0 & \sigma^{\mu} \\
\tilde{\sigma}^{\mu} & 0
\end{array}\right), \quad\left\{\Gamma^{\mu}, \Gamma^{\nu}\right\}=2 g^{\mu \nu},
$$

where indices $\alpha$ and $\beta$ - from the beginning of the Greek alphabet - are purely spinoric and $\mu$-from the middle - is a space index, and their nature is completely different.

Then the system (3.1) and the conjugated system can be written in the form

$$
\left[i \Gamma^{\mu} \partial_{\mu}-m I\right]_{\alpha}^{\beta} \psi_{\beta a}(x)=0, \quad \text { and } \quad \bar{\psi}_{a \beta}(x)\left[i \Gamma^{\mu} \overleftarrow{\partial}_{\mu}+m I\right]_{\alpha}^{\beta}=0
$$

These equations and all the conservation laws can be obtained from the Lagrangian

$$
L=\frac{1}{2} \bar{\psi}^{a \alpha}\left(i \Gamma^{\mu} \partial_{\mu}-m I\right)_{\alpha}^{\beta} \psi_{\beta a}-\frac{1}{2} \bar{\psi}^{a \alpha}\left(i \Gamma^{\mu} \overleftarrow{\partial}_{\mu}+m I\right)_{\alpha}^{\beta} \psi_{\beta a}
$$

\section{GAUGE TRANSFORMATIONS}

An essential feature of equations (3.3) is the possible form of their gauge transformations. Alongside the usual phase transformations with the $U(1)$ group, there exist transformations with the gauge group $S U(2)$ :

$$
\psi(x) \rightarrow M \psi(x) M^{-1}, \quad \bar{\psi}(x) \rightarrow M \bar{\psi}(x) M^{-1},
$$

where matrix $M$ is

$$
M=\left(\begin{array}{cc}
u & v \\
-v^{*} & u^{*}
\end{array}\right) \in S U(2, \mathbb{C}) .
$$

To ensure that the equations of motion (3.3) and the Lagrangian (3.4) are invariant with respect to the local phase transformations (4.1), where $M=M(x)$, we will introduce the covariant derivative

$$
D_{\mu} \psi(x)=\partial_{\mu} \psi(x)-i g\left[H_{\mu}(x), \psi(x)\right],
$$

where $H_{\mu}(x)$ is a $\omega$ vector with respect to the transformations $S O(5,1)$ with the matrix components from $S U(2)$.

$$
\left[H_{\mu}, \psi\right] \equiv H_{\mu} \cdot I \psi-I \psi \cdot H_{\mu} \equiv\left(\begin{array}{cc}
{\left[H_{\mu}, \varphi_{A}\right]} & 0 \\
0 & {\left[H_{\mu}, \chi^{\dot{A}}\right]}
\end{array}\right)
$$


On the possible existence of new fermionic degrees of freedom in $D=6159$

whose transformation obeys the law

$$
H_{\mu}(x) \rightarrow M(x) H_{\mu}(x) M^{-1}(x)-\frac{i}{g} \frac{\partial M(x)}{\partial x^{\mu}} M^{-1}(x) .
$$

Then $D_{\mu} \psi(x) \rightarrow M(x)\left(D_{\mu} \psi(x)\right) M^{-1}(x)$.

The commutator of the two covariant derivatives determines the tension tensor of the gauge field $H_{\mu}$ :

$$
\left[D_{\mu}, D_{\nu}\right] \psi=\left[F_{\mu \nu}, \psi\right]
$$

whose transformation is similar to that of the covariant derivative:

$$
F_{\mu \nu}(x) \rightarrow M(x) F_{\mu \nu}(x) M^{-1}(x)
$$

Note that a direct generalization of the usual unitary phase transformations of the Dirac bispinor

$$
\psi(x) \rightarrow U(x) \psi(x), \quad \bar{\psi}(x) \rightarrow \bar{\psi}(x) U^{+}(x)
$$

does not exhaust all possible phase transformations.

Phase transformations, indeed, are an automorphism of each component. The components of the bispinor $\psi(x)$ are complex matrices from algebra $M(2, \mathbb{C})$. Since any automorphism of algebra $M(n, \mathbb{C})$ is internal, i.e.

$$
\forall h: M(n, \mathbb{C}) \rightarrow M(n, \mathbb{C}), \exists g \in G L(n, \mathbb{C}): h(x)=g x g^{-1}, \forall x \in M(n, \mathbb{C})
$$

for any matrix $U(x)$ from (4.4) will match an $M(x)$ from (4.1), for which

$$
U(x) \psi(x)=M(x) \psi(x) M^{-1}(x)
$$

The opposite is not true.

The coordination of the field and quantum formalisms requires the invariance of commutational and anticommutational relations regarding the gauge transformations. It is possible to make sure that the commutators

$$
[\langle\psi(x), \psi(y)\rangle, \psi(z)]=i[S(x-z) \beta] \psi(y)-i[S(y-z) \beta] \psi(x)
$$

where the matrix $S(x)$ obeys the equation $\left(i \Gamma^{\mu} \partial_{\mu}-m\right) S(x)=0$ with the initial condition $\left.S(x)\right|_{t=0}=i \Gamma^{0} \delta(\vec{x}), \beta$ is the Dirac matrix $\Gamma^{0}$ and $\left\langle q_{1}, q_{2}\right\rangle \equiv$ $1 / 2\left(q_{1} \bar{q}_{2}-q_{2} \bar{q}_{1}\right)$ is a scalar product of quaternionic fields, where $\bar{q}$ is quaternionic conjugation. The commutation relations (4.5) are invariant with respect to the phase transformations (4.1) and noninvariant with respect to the transformations (4.4). 
The full Lagrangian of the Dirac and gauge fields will acquire the form

$$
L=\frac{1}{4} \operatorname{tr}\left[\bar{\psi}\left(i \Gamma^{\mu} D_{\mu}-m\right) \psi-\bar{\psi}\left(i \Gamma^{\mu} \overleftarrow{D}_{\mu}+m\right) \psi-F_{\mu \nu} F^{\mu \nu}\right]
$$

where

$$
\bar{\psi} \overleftarrow{D}_{\mu} \equiv \partial_{\mu} \bar{\psi}+i g\left[\bar{\psi}, H_{\mu}^{+}\right], F_{\mu \nu}=T_{a} \cdot F_{\mu \nu}^{a}
$$

and the generators of the group $S U(2)-T_{a}$ obey the correlation $\operatorname{tr} T_{a} T_{b}=$ $\frac{1}{2} \delta_{a b}$.

\section{QUATERNIONIC SUPERPARTICLE}

The superparticle action in the terms of $\mathbb{H}$-spinors corresponding to the BrinkSchwarz superparticle is

$$
S=\int d \tau \operatorname{tr}\left\{\left[\dot{X}_{A \dot{A}}-\frac{i}{2}\left(\psi_{A} \dot{\bar{\psi}}_{\dot{A}}-\dot{\psi}_{A} \bar{\psi}_{\dot{A}}\right)-\frac{1}{2} V P_{A \dot{A}}\right] \tilde{P}^{\dot{A} A}\right\}
$$

where $\psi_{A}$ and $\bar{\psi}_{\dot{A}}$ are $S L(2, \mathbb{H})$ spinors $\left(\bar{\psi}_{\dot{A}} \equiv\left(\psi_{A}\right)^{*}\right)$, where ${ }^{*}$ denotes the quaternion hermitian conjugation, $P_{A \dot{A}}$ is an $S L(2, \mathbb{H})$ hermitian vector.

It is easy to show that the action $(5.1)$ in the $S L(2, \mathbb{H})$ formalism has:

- global space-time supersymmetry

$$
\delta \psi_{A}=\varepsilon_{A}, \quad \delta P_{A \dot{A}}=\delta V=0, \quad \delta X_{A \dot{A}}=\frac{i}{2}\left(\varepsilon_{A} \bar{\psi}_{\dot{A}}-\psi_{A} \bar{\varepsilon}_{\dot{A}}\right)
$$

where $\varepsilon^{A}$ and $\varepsilon_{A}$ are $\tau$-independent Weyl and $S L(2, \mathbb{H})$ spinor parameters.

- reparametrisation invariance

$$
\delta \psi_{A}=\delta P_{A \dot{A}}=0, \quad \delta X_{A \dot{A}}=\xi(\tau) P_{A \dot{A}}, \quad \delta V=\dot{\xi}(\tau)
$$

where $\xi(\tau)$ is a $\tau$-dependent real parameter;

- local fermionic gauge invariance

$$
\begin{gathered}
\delta \psi_{A}=P_{A \dot{A}} \eta^{\dot{A}}, \quad \delta P_{A \dot{A}}=0, \quad \delta X_{A \dot{A}}=\frac{i}{2}\left(\psi_{A} \bar{\eta}^{B} P_{B \dot{A}}-P_{A \dot{B}} \eta^{\dot{B}} \bar{\psi}_{\dot{A}}\right) \\
\delta V=i\left(\dot{\psi}_{A} \bar{\eta}^{A}-\eta^{\dot{A}} \dot{\bar{\psi}}_{\dot{A}}\right)
\end{gathered}
$$

where $\eta^{a}(\tau)$ and $\eta^{\dot{A}}(\tau)$ are $\tau$-dependent Weyl and $S L(2, \mathbb{H})$ spinor.

The equations of motion and constraints derived from action (5.1) are

$$
\operatorname{tr} P_{A \dot{A}} \tilde{P}^{\dot{A} A}=0, \quad \dot{P}_{A \dot{A}}=0, \quad \tilde{P}^{\dot{A} A} \dot{\psi}_{A}=0
$$


On the possible existence of new fermionic degrees of freedom in $D=6161$

$$
P_{A \dot{A}}=\frac{1}{V}\left[\dot{X}_{A \dot{A}}-\frac{i}{2}\left(\psi_{A} \dot{\bar{\psi}}_{\dot{A}}-\dot{\psi}_{A} \bar{\psi}_{\dot{A}}\right)\right]
$$

We note that the first two equations of (5.4) imply

$$
P_{A \dot{A}}=\pi_{A} \bar{\pi}_{\dot{A}}
$$

where $\pi_{A}$ is a $\tau$-independent $S L(2, \mathbb{H})$ spinor. Using this expression and other equations of (5.4), the most general solution of the classical equations of motion is

$$
X_{A \dot{A}}(\tau)=X_{A \dot{A}}^{0}+\alpha(\tau) \pi_{A} \bar{\pi}_{\dot{A}}+\frac{i}{2}\left[\psi_{A}^{0} u^{*}(\tau) \bar{\pi}_{\dot{A}}-\pi_{A} u(\tau) \bar{\psi}_{\dot{A}}^{0}\right]
$$

where $\alpha(\tau)=\int d \tau\left\{\frac{i}{2}\left[u(\tau) \dot{u}(\tau)^{*}(\tau)-\dot{u}(\tau) u^{*}(\tau)+V(\tau)\right]\right\}$.

\section{PARASTATISTICS AND EXTENDED OBJECT}

As follows from commutation relation (4.5), quaternionic spinors obey the paracommutation relation but not the ordinary commutation relation. This difference leads to essential statistical properties of $\mathbb{H}$-fermions.

It is known that in the framework of Wightman axioms in higher dimensions all particles are either fermions or bosons. This general remark coincides with a strong theoretical analysis of quantum field theory axiom, which has the status of "no-go" theorem for free relativistic particles with fractional statistics in $D=4[4 ; 14]$.

Such a strong theoretical remark seems to exclude all attempts to consider quons in a higher-dimensional space-time. However, we can overcome this problem by considering a non-local relativistic object in $D=4$ and moredimensional space-time. Lately such objects are being intensively studied in high energy physics as unification models of all interactions. These objects are superstrings and super- $p$-branes $[9 ; 16]$.

A straightforward introduction of quonic string and $p$-brane evokes an immediate contradiction. Indeed, if we replace the fermionic degrees of freedom by quonic ones, we obtain something like a quonic string, but after Fourier transformations under space coordinate we may consider the string as a set of local quonic fields whose existence is forbidden by local quantum field theory axioms.

In $D=6$ space-time, let us use the geometrical Poincaré duality. Indeed, the 4-brane is dual regarding the string. If a usual superstring is expressed in terms of quaternions, because of the string linearity we shall immediately obtain a set of local quaternionic $\mathbb{H}$-fields obeying the paracommutational correlations, however, this is forbidden by Wightman's axioms. 
At the same time, if a super-4-brane with the same degrees of freedom and gauge group are expressed in terms of $\mathbb{H}$-fields, then, by force of the essential nonlinearity of the $p$-brane we will obtain a relativistic object obeying the paracommutational correlations, however, in this case nonlinear and extended, and this is not forbidden by quantum field theory axioms.

Thus, we see that the main differences are reduced to the quantum case. Unfortunately, there are considerably less reports on the quantization of $\mathbb{H}-$ than $\mathbb{C}$-fields.

Which unusual properties of $\mathbb{H}$-branes can be expected to manifest?

We will strictly differentiate between parastatistics and fractional statistics.

Recently, the para-supersymmetric generalization of supersymmetric quantum mechanics (PSUSY) has been developed $[1 ; 2]$. The simplest example of PSUSY quantum-mechanical Hamiltonian of the order 3, which describes a spin-1 particle, is realized as $Q^{3}=Q H$ with $Q^{2} \neq H$, where $Q$ is a conserved para-supercharge [13]. In general, a PSUSY quantum-mechanical Hamiltonian of the order $M$ is of the form $Q^{M}=Q^{M-2} H+\ldots$ and describes a spin $(M-1) / 2$ particle [14]. Otherwise, there is an alternative generalization of SUSY quantum mechanics which we call fractional supersymmetric quantum mechanics (FSUSY) of the order $F[5 ; 6]$. Hamiltonians of this new kind are expressed as the $F^{t h}$ power of a conserved fractional supercharge: $Q^{F}=H$ with $F=2,3, \ldots$. This means that FSUSY transformations are the $F^{t h}$ roots of time translation.

In general, we have the following properties of the harmonic oscillator for the FSUSY cases of arbitrary order. In the case when FSUSY is not spontaneously broken, i.e. the ground state is invariant under FSUSY transformations since $Q|0\rangle=0$, the spectrum is $F$-fold degenerate. The ground state is one or $(F-1)$-fold degenerate depending on the kind of FSUSY. The main difference is the negative value of ground state energy in PSUSY. Note here that in the case of quaternionic super 4 -brane in $D=6$ space-time we have a set of interacting harmonic oscillators, and the negativity of spectrum and the numbers of degenerations must be verified.

\section{CONCLUSIONS AND DISCUSSION}

The existence of the new degrees of freedom in $D=6$ and $D=10$ leads to the following consequences.

Firstly, a new light is thrown on the origin of unitary symmetries: $S U(2)$ and $S U(3)$ in $D=4$ could be treated as the residual symmetries from the gauge fields in $D=6$ and $D=10$. Moreover, we cannot, at least at the first glance, exclude the Kaluza-Klein origin of gauge symmetry in $D=6$ and $D=4$.

Secondly, the equality of the bosonic, $\mathbb{C}$-fermionic and $\mathbb{H}$-fermionic degrees 
On the possible existence of new fermionic degrees of freedom in $D=6163$

of freedom in $D=6$ implies a $\mathbb{H}$-hypersymmetry - a generalization of usual symmetry on the quaternionic bispinors and $\mathbb{O}$-hypersymmetry in $D=10$.

Thirdly, it becomes reasonable to suggest that compactification $\mathcal{M}^{10} \rightarrow \mathcal{M}^{4}$ occurs via the intermediate dimension $\mathcal{M}^{6}: \mathcal{M}^{10} \rightarrow \mathcal{M}^{6} \rightarrow \mathcal{M}^{4}$.

A schematic representation of the suggested picture could be as follows:

$$
\begin{array}{lcccc}
\text { Dimension } D & 3 & 4 & 6 & 10 \\
& & & & \\
\text { Lorentz group } S O(2,1) & \leftarrow S O(3,1) & \leftarrow S O(5,1) & \leftarrow S O(9,1) \\
& \downarrow & \downarrow & \downarrow & \downarrow \\
\text { Spin group } & S L(2, \mathbb{R}) & \leftarrow S L(2, \mathbb{C}) & \leftarrow S L(2, \mathbb{H}) & \leftarrow S L(2, \mathbb{O})
\end{array}
$$

The next step could be elaboration of a non-contradictory hypersymmetrical string model in $D=10$.

\section{REFERENCES}

[1] J. Beckers and N. Debergh. On parasupersymmetry and remarkable Lie structures. $J$. Phys. A: Math. and Gen., 23, L751S-L955S, 1991.

[2] K. Behrndt and G. Dall'Agata. Vacua of $N=2$ gauged supergravity derived from non-homogeneous quaternionic spaces. Nucl.Phys.B, 627, 357-380, 2002.

[3] P. Casteill, E. Ivanov and G. Valent. $U(1) \times U(1)$ Quaternionic Metrics from Harmonic Superspace. Nucl.Phys. B, 627, 403-444, 2002.

[4] C.K. Chow and O.W. Greenberg. Quons in Relativistic Theories Must be Bosons or Fermions. Phys. Lett. A, 283, 20-24, 2001.

[5] S. Durand. Fractional Superspace Formulation of Generalized Super-Virasoro Algebras. Mod. Phys. Lett. A, 7, 2905-2912, 1992.

[6] S. Durand. Fractional supersymmetry and quantum mechanics. Phys. Lett. B., 312, 115-120, 1993.

[7] S. Fubini and H. Nicolai. The octonionic instantion. Phys. Lett. B., 155, 369-383, 1985.

[8] I.A. Harvey and A. Strominger. Octonionic superstring solitons. Phys. Lett. B., 66, $549-553,1991$.

[9] M. Kaku. Introduction to Superstrings and M-theory. Springer, New York-London, 2-nd Ed., 1999.

[10] T. Kimura and I. Oda. Superparticles and division algebras. Progr. Theor. Phys., 80, $1-6,1988$.

[11] T. Kugo and P. Taunsend. Nucl. Phys. B, volume 221. 1983.

[12] S.de Leo and G. Scolarici. Right eigenvalue equation in quaternionic quantum mechanics. J. Phys. A, 33, 2971-2995, 2000.

[13] M.D. Maia and V.B. Bezerra. Geometric Phase in Quaternionic Quantum Mechanics. Int. J. Theor. Phys., 40, 1283-1294, 2001.

[14] J. Mund. No-go theorem for free relativistic anyons in $d=2+1$. Lett. Math. Phys., 43, 319-328, 1998.

[15] I. Oda, T. Kimura and A. Nakamura. Ten dimensions and octonious. Progr. Theor. Phys., 80, 367-372, 1988.

[16] J. Polchinski. String Theory, volume 1. Cabridge University Press, UK London, 1999. 
[17] A. Razon, L.P. Horwitz and L.C. Biedenharn. On a basic theorem of quaternion modules. J. Math. Phys., 30, 59-69, 1989.

[18] A. Sudbery. Division algebras, (pseudo)orthogonal groups and spinors. J. Phys. A: Math. and Gen., 17, 939-955, 1984.

[19] B.de Wit, M. Rocek and S. Vandoren. Gauging Isometries on Hyperkahler Cones and Quaternion-Kahler Manifolds. Phys. Lett. B, 511, 302-310, 2001.

Galimas naujų fermionių laisvès laipsnių egzistavimas erdvẻlaikyje $D=6$

\section{P. Miškinis}

Kvaternioninius bispinorius traktuodami kaip naujus fermioninius laukus $D=6$-mačiame erdvèlaikyje, gausime, kad kalibruotojo lauko bei supersimetrijos sąvokos gali būti iš esmès apibendrintos ir praplèstos. Pasiūlytas Brinko-Švarco kvaternioninès superdalelès modelis. Parodyta, kad išplèstiniai objektai su lokaline bei globaline supersimetrija turi tenkinti parastatistikos sąryšius. Trumpai aptartos galimos kosmologinio modelio pasiketimo pasekmès. 